\title{
MICROLENSING EVIDENCE THAT A TYPE 1 QUASAR IS VIEWED FACE-ON
}

\author{
Shawn Poindexter and Christopher S. KochaneK \\ Department of Astronomy and Center for Cosmology and AstroParticle Physics, The Ohio State University, 140 W 18th Ave, Columbus, OH 43210, USA; \\ sdp@astronomy.ohio-state.edu, ckochanek@astronomy.ohio-state.edu \\ Received 2009 October 19; accepted 2010 February 4; published 2010 March 4
}

\begin{abstract}
Using a microlensing analysis of 11 years of OGLE $V$-band photometry of the four image gravitational lens Q2237+0305, we measure the inclination $i$ of the accretion disk to be $\cos i>0.66$ at $68 \%$ confidence. Very edge on ( $\cos i<0.39$ ) solutions are ruled out at $95 \%$ confidence. We measure the $V$-band radius of the accretion disk, defined by the radius where the temperature matches the monitoring band photon emission, to be $R_{V}=5.8_{-2.3}^{+3.8} \times 10^{15} \mathrm{~cm}$ assuming a simple thin disk model and including the uncertainties in its inclination. The projected radiating area of the disk remains too large to be consistent with the observed flux for a $T \propto R^{-3 / 4}$ thin disk temperature profile. There is no strong correlation between the direction of motion (peculiar velocity) of the lens galaxy and the orientation of the disk.
\end{abstract}

Key words: gravitational lensing: micro - methods: numerical - quasars: individual (Q2237+0305)

Online-only material: color figures

\section{INTRODUCTION}

In the active galactic nucleus (AGN) unification model (e.g., Antonucci 1993; Urry \& Padovani 1995), orientation differences among intrinsically similar objects are thought to account for many of the different observational properties of AGNs. In particular, a dusty "torus" may frequently obscure the central engine from direct observation when viewed edge-on. The bright, Type 1 broad line quasars are thought to be viewed mostly face-on, so that the accretion disk is not obscured by material in the equatorial plane, and Type 2 narrow line quasars are viewed closer to edge-on through the obscuring material. Thus, if we could resolve the disk of a Type 1 quasar, we would expect it to be closer to face-on than edge-on. Unfortunately, familiar methods cannot resolve accretion disks, so we have only indirect measures of AGN disk orientation. For example, the projected axes of radio jets (Blandford \& Konigl 1979) and ionization cones (Elvis 2000) both support this picture. There are, however, no actual measurements of disk orientation.

While quasar accretion disks are too small to be resolved by direct imaging, gravitational microlensing provides a natural telescope to study the structure of quasar accretion disks and the properties of cosmologically distant lens galaxies where we see multiple images of background quasars (see Wambsganss 2006). In addition to the mean potential of the lens galaxy, each image is also magnified by the microlensing effects of the nearby stars. Since the observer, the lens galaxy and its stars, and the quasar are all moving, microlensing is observed as uncorrelated time variability in each of the quasar images. The amplitudes of these variations depend on the structure of the accretion disk and the properties of the lens galaxy.

Quasar microlensing is most sensitive to the projected area of the accretion disk relative to the source plane Einstein radius,

$$
\begin{aligned}
R_{\mathrm{E}} & =D_{\mathrm{OS}} \sqrt{\frac{4 G\langle M\rangle}{c^{2}} \frac{D_{\mathrm{LS}}}{D_{\mathrm{OL}} D_{\mathrm{OS}}}} \\
& =1.8 \times 10^{17}\left(\frac{\langle M\rangle}{M_{\odot}}\right)^{1 / 2} \mathrm{~cm},
\end{aligned}
$$

where $G$ is the gravitational constant, $c$ is the speed of light, $\langle M\rangle$ is the mean stellar mass of the stars, $D_{\mathrm{LS}}, D_{\mathrm{OL}}$, and $D_{\mathrm{OS}}$ are the angular diameter distances between the lens-source, observer-lens, and observer-source, respectively, and we have used the lens and source redshifts for Q2237+0305 (Q2237 hereafter, $z_{1}=0.0394, z_{\mathrm{s}}=1.685$; Huchra et al. 1985). The smaller the accretion disk, the higher the variability amplitude from microlensing. In general, the emission profile is difficult to determine, as models having similar half-light radii show similar microlensing variability (Mortonson et al. 2005). There has been little examination of other structural parameters of disks, except for Congdon et al. (2007), who demonstrated in simulations that the microlensing signal is sensitive to the ellipticity and orientation of the accretion disk. If detectable in practice, measuring the apparent ellipticities of accretion disks provides an important test of AGN unification models and opens the possibility of examining the complex effects of relativity on the apparent surface brightness of the disk (e.g., Hubeny et al. 2001).

Measurements of quasar disk sizes using microlensing are now common. Recent efforts have studied individual sizes (e.g., Morgan et al. 2008a), the relationships between size and wavelength (Anguita et al. 2008; Bate et al. 2008; Eigenbrod et al. 2008a; Poindexter et al. 2008; Floyd et al. 2009; Mosquera et al. 2009), size and black hole mass (Morgan et al. 2010), and the sizes of thermal and non-thermal emission regions (Pooley et al. 2007; Morgan et al. 2008b; Chartas et al. 2009; Dai et al. 2009). All these studies used circular accretion disks and static magnification patterns that neglect the random motion of stars in the lens galaxy.

Determining the shape and orientation of a disk depends on the correlations between the anisotropic structure of the disk and the anisotropic structure in the magnification patterns created by the shear (tidal gravity) local to each image (see Figure 1; Congdon et al. 2007). Existing microlensing studies cannot safely explore these issues because they neglect the motions of the stars in the lens galaxy and use "static" magnification patterns. Since the stellar velocity dispersions of lens galaxies are comparable to the peculiar velocities of galaxies, the patterns 


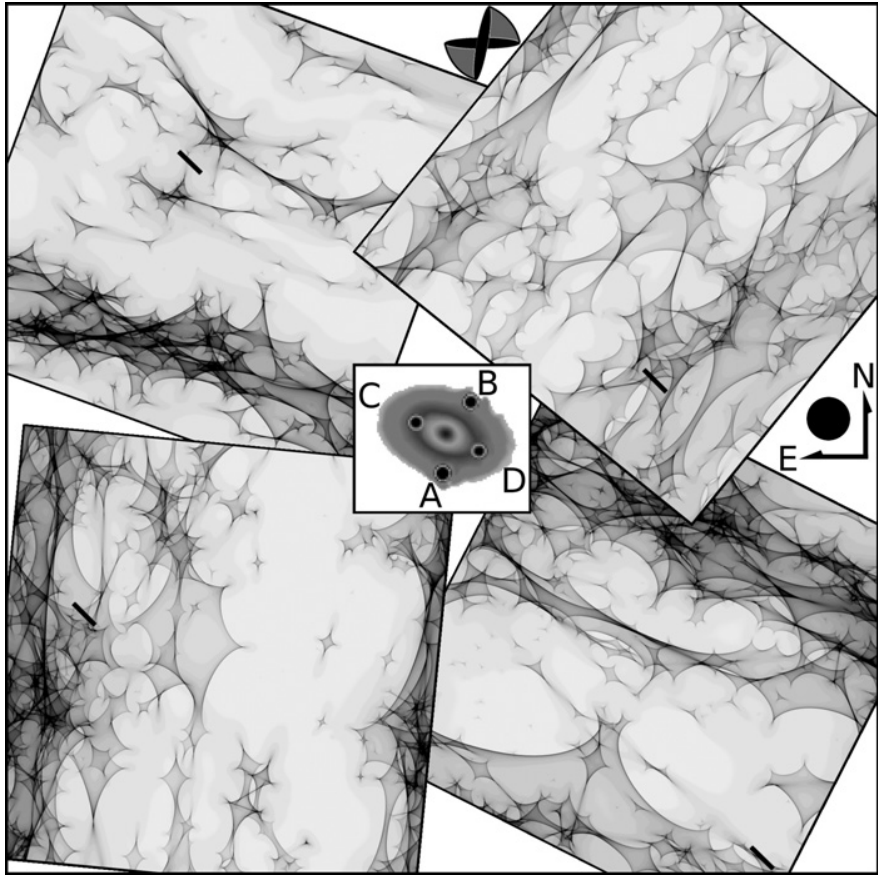

Figure 1. Example of a trial source trajectory (dark line segments) superposed on instantaneous point-source magnification patterns for $\left\langle M / M_{\odot}\right\rangle=0.3$. Darker shades indicate higher magnification. An $H S T H$-band image in the center labels the images and the corresponding magnification patterns. Each pattern is rotated to have the correct orientation relative to the lens. This particular LC2 trial has an effective lens-plane velocity of $\sim 600 \mathrm{~km} \mathrm{~s}^{-1}$ northeast. The "bow tie" (top) exhibits the two peaks in the major-axis position angle (P.A.) distribution together with the shaded region that approximately represents the $68 \%$ confidence region. The solid disk (right) has a radius of $10^{17} \mathrm{~cm}$.

change on the same timescale as the source traverses them. Ignoring these stellar motions will overestimate the coherence of the magnification patterns (see Wyithe et al. 2000a; Kochanek et al. 2007) and likely render estimates of disk shapes unrealistic. With a few exceptions that do not focus on disk structure (see Paper I and Wyithe et al. 1999), analyses of microlensing data have used static magnification patterns because of the computational challenges. In Paper I (Poindexter \& Kochanek 2010), we solved these computational problems and examined the peculiar velocity of the lens galaxy of Q2237 and the mean mass of its stars. In this paper, we measure the size, inclination, and position angle (P.A.) of the accretion disk in Q2237. In Section 2, we describe the data set used for our microlensing analysis, our disk model, and outline our overall approach. Our results are presented in Section 3, and a discussion follows in Section 4.

\section{DATA AND METHODS}

We analyze the nearly 11 years of Optical Gravitational Lensing Experiment (OGLE) $V$-band photometric monitoring data for Q2237 (Udalski et al. 2006). To speed our analysis and as a cross check on the results, we divided the data into two separate light curves. The first light curve (LC1 hereafter) ranges from JD 2450,663 to JD 2452,621 and consists of 100 epochs. The second light curve (LC2 hereafter) has 230 epochs from JD 2452,763 to JD 2454,602. Each light curve covers just over five years. We broaden the OGLE uncertainties by our estimate of the systematic uncertainties in the photometry of $0.02,0.03,0.04$, and $0.05 \mathrm{mag}$ in quadrature (Poindexter \& Kochanek 2010).
We analyze these light curves using the Bayesian Monte Carlo method of Kochanek (2004), expanded to include motions of stars as detailed in Paper I. For each epoch of the light curve, we generate a magnification pattern including the random motion of the stars. We used fixed mean masses of $\langle M\rangle=$ $0.01,0.03,0.1,0.3,1,3$, and $10 M_{\odot}$, and a mass function of $d N / d M \propto M^{-1.3}$ with a dynamic range $M_{\max } / M_{\min }=50$ based on Gould (2000). In Paper I, we find that the best fit to the data is for $\langle M\rangle=0.3 M_{\odot}$. The stars are assigned a random velocity in each coordinate from a Gaussian distribution with $\sigma=170 \mathrm{~km} \mathrm{~s}^{-1}$ based on the observed velocity dispersion (G. van de Ven 2009, personal communication; Trott et al. 2010). The orbit of Earth (parallax effect) and the rotation of the lens galaxy are both included in the simulation. We convolve the patterns with the disk models described in Section 2. We draw the bulk velocities of the observer, lens galaxy, and source from a Gaussian of dispersion $\sigma=1000 \mathrm{~km} \mathrm{~s}^{-1}$ on the lens plane in each coordinate. We later reweight the results to a more compact velocity prior based on the projection of the CMB dipole velocity (Hinshaw et al. 2009) onto the lens plane, which is small $(-50,-23) \mathrm{km} \mathrm{s}^{-1}$, and the (one-dimensional) peculiar velocity dispersions of the lens and source of $327 \mathrm{~km} \mathrm{~s}^{-1}$ and $230 \mathrm{~km} \mathrm{~s}^{-1}$ (estimated from J. L. Tinker, 2010, in preparation), respectively. Because of the low lens redshift and the small projected dipole, the peculiar velocity of the lens is by far the most important factor (see Paper I). We then randomly draw light curves for each image and fit them to the data. Bayes theorem is used to combine the goodness of fit for the trials as measured by a $\chi^{2}$ statistic into probability distributions for each variable of interest. These procedures are described in detail in Kochanek (2004) and Paper I.

\subsection{Accretion Disk Model}

We employ a generic thin disk model for which the surface temperature scales as $T \propto R^{-3 / 4}$, with $R$ being the radius (Shakura \& Sunyaev 1973). The microlensing signal is primarily sensitive to the half-light radius of the disk (Mortonson et al. 2005), which controls the effective smoothing area of the disk, and, to date, studies have been unable to distinguish differing radial profiles (e.g., Kochanek 2004). A more realistic disk model would include a central hole whose size depends on the last stable orbit and general relativistic effects modify the underlying intensity profiles (e.g., Hubeny et al. 2001). We have not presently pursued these features because they add many model parameters, and because it is unclear whether the temperature profile of the thin disk model is correct. In particular, microlensing studies suggest the need for a flatter temperature profile (e.g., Pooley et al. 2007; Morgan et al. 2010; Poindexter et al. 2008; Eigenbrod et al. 2008a) in order to reconcile the microlensing sizes with the observed optical fluxes. Such changes in the temperature profile have also been suggested to explain the deviations of the observed spectral slope from the thin disk emission (see the reviews by Koratkar \& Blaes 1999 and Blaes 2004).

Thus, we assume that the face-on $(\cos i=1)$ surface brightness of the disk is

$$
f_{\nu}=\frac{2 h c}{\lambda_{\text {rest }}^{3}}\left[\exp \left(R / R_{\lambda}\right)^{3 / 4}-1\right]^{-1}
$$


where

$$
\begin{aligned}
R_{\lambda}= & {\left[\frac{45 G \lambda_{\text {rest }}^{4} M_{\mathrm{BH}} \dot{M}}{16 \pi^{6} h c^{2}}\right]^{1 / 3} } \\
= & 9.7 \times 10^{15}\left(\frac{\lambda_{\text {rest }}}{\mu \mathrm{m}}\right)^{4 / 3} \\
& \times\left(\frac{M_{\mathrm{BH}}}{10^{9} M_{\odot}}\right)^{2 / 3}\left(\frac{L}{\eta L_{E}}\right)^{1 / 3} \mathrm{~cm}
\end{aligned}
$$

corresponds to the radius where the disk temperature equals the photon energy, $k T=h c / \lambda_{\text {rest }}, M_{\mathrm{BH}}$ is the black hole mass, $\dot{M}$ is the accretion rate, $L / L_{\mathrm{E}}$ is the luminosity relative to the Eddington luminosity, and $\eta=L /\left(\dot{M} c^{2}\right)$ is the radiative efficiency of the accretion disk. The half-light radius of the disk is $R_{1 / 2}=2.44 R_{\lambda}$. We now simply treat the disk as an infinitely thin disk viewed at inclination angles $i$, selected from a uniform distribution in $\cos i=0.2,0.4,0.6,0.8$, and 1.0 (faceon). We include no relationship between the surface brightness and the viewing angle other than this simple projection effect in this first exploration of the problem. For full relativistic disk models, there are many complexities such as Doppler shifts, redshifts, and bending of ray trajectories (e.g., Agol 1997), but the bulk of the optical flux comes from relatively large radii, $R_{V} / R_{\mathrm{g}} \sim 30$ for $R_{\mathrm{g}}=G M_{\mathrm{BH}} / c^{2}=2 \times 10^{14} \mathrm{~cm}$, where these effects are less important. Here we use Morgan et al.'s (2010) estimated black hole mass of $1.3 \times 10^{9} M_{\odot}$ found by applying the virial relation of Vestergaard \& Peterson (2006) to the CIV line width measurement from Yee \& De Robertis (1991). Since the orientation of the projected disk relative to the magnification patterns also affects the results, we considered 18 major axis P.As from $0^{\circ}$ to $170^{\circ}$ in steps of $10^{\circ}$, where the remaining angles are covered by the reflection symmetry of the disk model. We parameterized the size of the disk by the projected area, $\pi R_{V}^{2} \cos i$, as this is likely to have less correlation with the inclination angle because it keeps the projected area of the disk constant.

We must also worry about whether all the observed emission arises directly from the accretion disk. The light curves can be contaminated by broad line emission on much larger scales or some of the emission from the disk can be scattered on larger scales (see Dai et al. 2009; Morgan et al. 2008b, 2010). At $V$-band, the contamination from broad line emission, mainly Fe pseudocontinuum emission and C III] $\lambda 1909$, contributes of order $20 \%$ of the flux in the spectral models of Eigenbrod et al. (2008b). To examine the effect of this dilution, we ran models with $0 \%, 20 \%$, and $40 \%$ contamination by emission on large, unmicrolensed scales for the $\langle M\rangle=0.3 M_{\odot}$ case. Adding an unmicrolensed contamination fraction, $f$, in the $V$ band also decreases the flux size estimate by $(1-f)^{1 / 2}$ unless the contamination is due to scattering of the disk emission on large scales (see the discussion in Morgan et al. 2010).

\section{RESULTS}

We estimate the projected area of the accretion disk $\pi R_{V}^{2} \cos i$, the deprojected radius $R_{V}$, the disk inclination $i$, and the major axis P.A. We also do a limited set of tests with different amounts of unmicrolensed flux that may influence our analysis. We quote the results from the combined analysis of LC1 and LC2, but also show the results from the independent analyses of LC1 and LC2. Since the results are always mutually consistent, we only report quantitative results for the combined analysis.

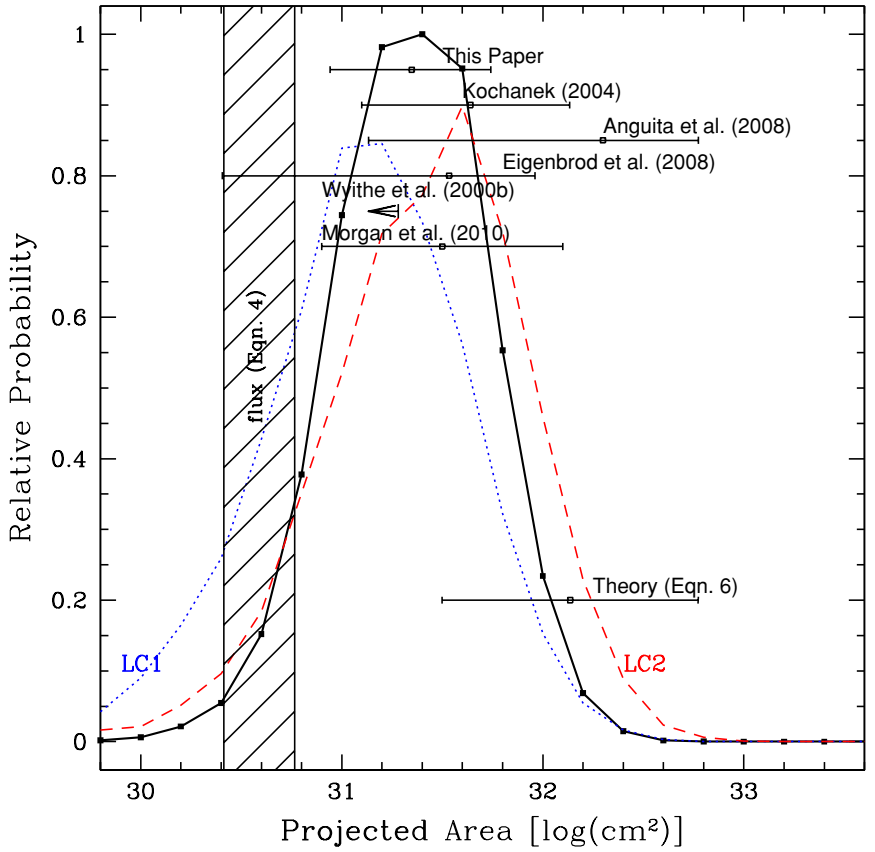

Figure 2. Projected area $\pi R_{V}^{2} \cos i$ distribution of the accretion disk. The blue dotted (red dashed) curve shows the results from the analysis of only LC1 (LC2). The black curve is the combined result from both LC1 and LC2. The horizontal bars compare $68 \%$ confidence regions from earlier studies assuming $\langle M\rangle=0.3 M_{\odot}$ for estimates which depended on the mean mass. The upper limit found by Wyithe et al. (2000b) is at 99\% confidence. The "flux" estimate is the area predicted based on the observed flux (Equation (4)) and is independent of inclination. The "theory" estimate is the area thin disk theory predicts assuming $\cos i=1$, based on the estimated black hole mass for Q2237 (Equation (6)).

(A color version of this figure is available in the online journal.)

We find (Figure 2) the projected $V$-band area of the quasar defined by $\pi R_{V}^{2} \cos i$ to be $8.7 \times 10^{30} \mathrm{~cm}^{2}<\pi R_{V}^{2} \cos i<$ $5.5 \times 10^{31} \mathrm{~cm}^{2}\left(4.7 \times 10^{30} \mathrm{~cm}^{2}<\pi R_{V}^{2} \cos i<9.4 \times 10^{31} \mathrm{~cm}^{2}\right)$ at $68 \%(95 \%)$ confidence with a median of $\pi R_{V}^{2} \cos i=$ $2.2 \times 10^{31} \mathrm{~cm}^{2}$, where the size scale $R_{V}$ is defined by Equation (3). After de-projecting the area based on each trial's inclination, we find that the $V$-band radius of the accretion disk is $3.5 \times 10^{15} \mathrm{~cm}<R_{V}<1.0 \times 10^{16} \mathrm{~cm}\left(2.6 \times 10^{15} \mathrm{~cm}<\right.$ $\left.R_{V}<1.4 \times 10^{16} \mathrm{~cm}\right)$ at $68 \%(95 \%)$ confidence with a median value of $R_{V}=5.8 \times 10^{15} \mathrm{~cm}$ (Figure 3 ). This is consistent with our earlier results in Kochanek (2004) and Morgan et al. (2010), of $R_{V}=3.7_{-1.7}^{+2.9} \times 10^{15} / \sqrt{\cos i} \mathrm{~cm}$ and $R_{V}=$ $3.2_{-1.6}^{+3.1} \times 10^{15} / \sqrt{\cos i} \mathrm{~cm}$ using the same method without dynamic patterns, a smaller velocity prior, and shorter light curves. Other analyses by Wyithe et al. (2000b), Yonehara (2001), Vakulik et al. (2007), Anguita et al. (2008), and Eigenbrod et al. (2008a) have found generally consistent results of $R_{V}<2.5 \times 10^{15} \mathrm{~cm}$ (at $99 \%$ confidence), $R_{V} \lesssim 1.8 \times 10^{17} \mathrm{~cm}$, $R_{V} \sim 2 \times 10^{15} \mathrm{~cm}, R_{V}=8.0_{-5.9}^{+5.7} \times 10^{15} \sqrt{M / 0.3 M_{\odot}} \mathrm{cm}$, and $R_{V}=3.3_{-2.4}^{+2.1} \times 10^{15} \mathrm{~cm}$ (see Figure 3), but using less data and with far stronger systematic assumptions. These estimates are all for $\cos i=1$ and should scale as $1 / \sqrt{\cos i}$. If we compare projected areas (Figure 2), then the comparisons are (to first order) independent of the inclination angle; if we compare disk scale lengths, there will be an inclination angle dependence. While generally consistent with our results, our calculations use more data and are considerably more realistic, making it difficult to evaluate differences, especially the uncertainties. 


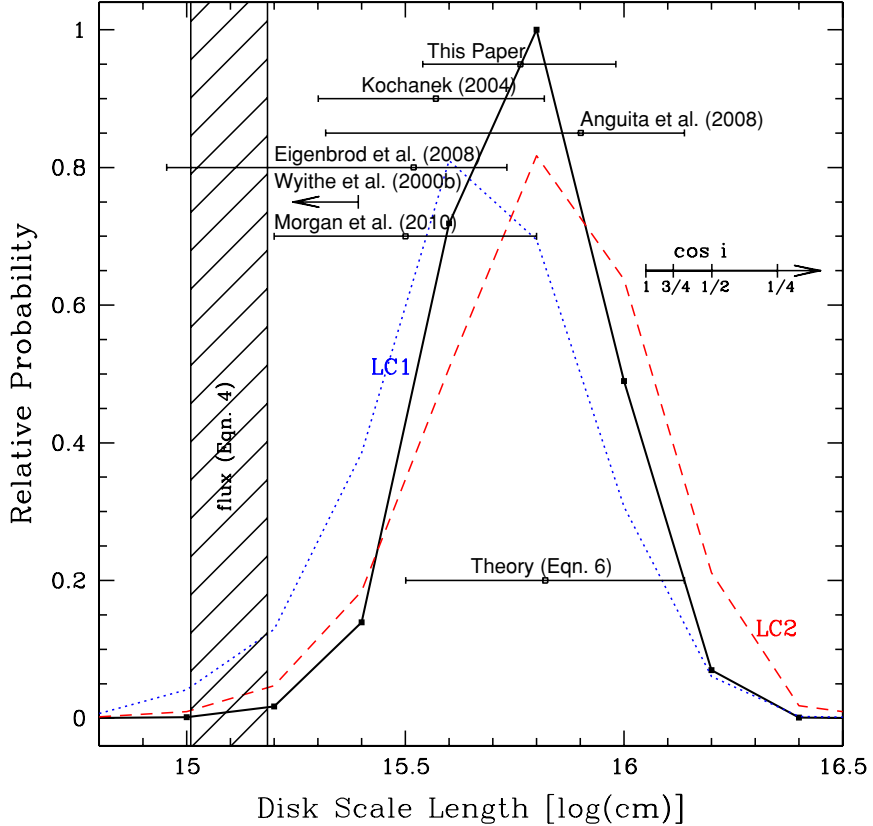

Figure 3. Disk scale length $R_{V}$ after de-projection from the joint analysis of LC1 and LC2. The blue dotted (red dashed) curve shows the results from the analysis of only $\mathrm{LC} 1$ (LC2). The earlier results are shown assuming $\cos i=1$ for these face-on models. A scale on the right shows how they would shift to larger sizes if $\cos i<1$. The flux size has the same inclination dependence as the earlier results, while the theory size is independent of inclination. Our test with $20 \%$ (40\%) of light being contamination emitted on much larger scales resulted in a $26 \%(55 \%)$ smaller radius.

(A color version of this figure is available in the online journal.)

We can compare our measurement to those predicted by the observed fluxes or thin disk theory. If we assume only the $T \propto R^{-3 / 4}$ temperature profile of a thin disk model (Shakura \& Sunyaev 1973), where we can ignore the inner disk edge for these wavelengths, then the observed flux constrains the disk size by matching the integrated flux from a disk with the emission profile of Equation (3) to the observed flux. We estimate that the magnification corrected $I$-band flux is $I=18.03 \pm 0.44 \mathrm{mag}$, and this corresponds to a disk radius of

$$
\begin{aligned}
R_{V}= & 1.7 \times 10^{15} \frac{1}{\sqrt{\cos i}}\left(\frac{D_{\mathrm{OS}}}{r_{\mathrm{H}}}\right) \\
& \times\left(\frac{\lambda_{\mathrm{I}, \mathrm{obs}}}{\mu \mathrm{m}}\right)^{3 / 2} 10^{-0.2(I-19)} h^{-1} \mathrm{~cm},
\end{aligned}
$$

where $D_{\mathrm{OS}} / r_{\mathrm{H}}$ is the angular diameter distance to the quasar relative to the Hubble radius, $r_{\mathrm{H}}$ (see Morgan et al. 2010). This gives $\pi R_{V}^{2} \cos i=3.9_{-1.3}^{+1.9} \times 10^{30} \mathrm{~cm}$. Assuming our best-fit inclination, $\cos i=0.8, R_{V}=1.25_{-0.23}^{+0.28} \times 10^{15} \mathrm{~cm}$.

Agol et al. (2009) estimate that the bolometric luminosity of the quasar is $L_{\mathrm{Agol}}=4 \times 10^{46} \mathrm{erg} \mathrm{s}^{-1}$, which corresponds to an Eddington factor of

$$
\frac{L}{L_{E}}=\frac{1}{3} \frac{L_{\mathrm{bol}}}{L_{\mathrm{Agol}}} \frac{10^{9} M_{\odot}}{M_{\mathrm{BH}}},
$$

that is typical of luminous quasars (Kollmeier et al. 2006). If we use this to replace the $L / L_{E}$ factor in Equation (3), the size estimate becomes

$$
R_{\lambda}=6.6 \times 10^{15}\left(\frac{\lambda_{\text {rest }}}{\mu \mathrm{m}}\right)^{4 / 3}\left(\frac{M_{\mathrm{BH}}}{10^{9} M_{\odot}} \frac{L_{\text {bol }}}{L_{\mathrm{Agol}}} \frac{1}{\eta}\right)^{1 / 3} \mathrm{~cm},
$$

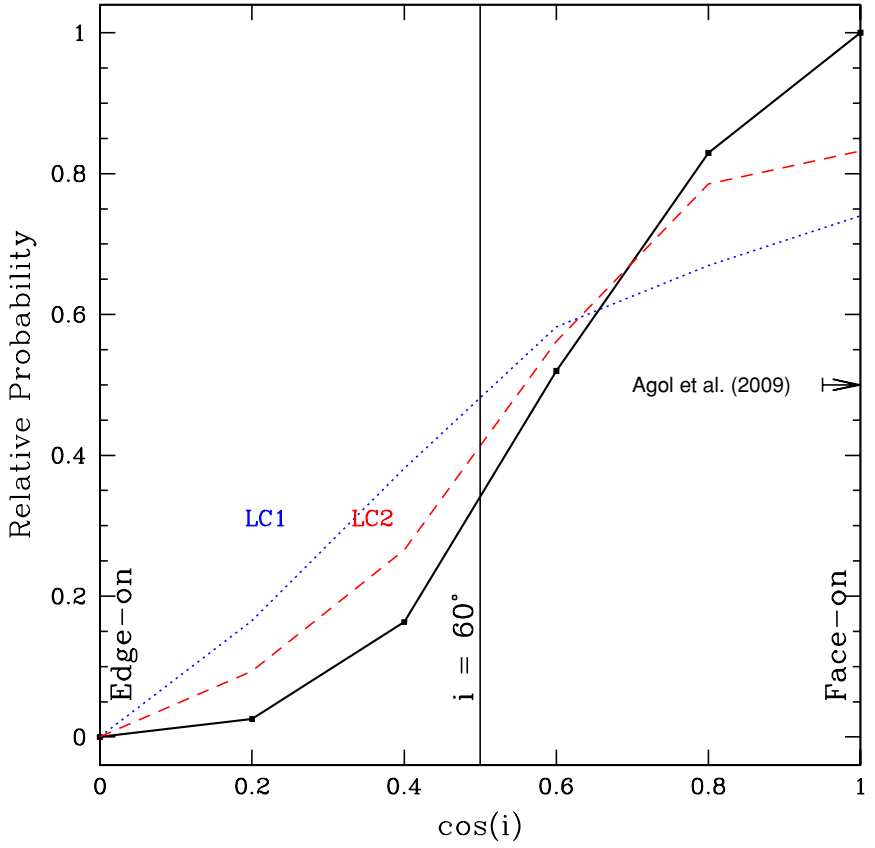

Figure 4. Cosine of the disk inclination ( $\cos i=1.0$ is face-on). The blue dotted (red dashed) curve shows the results from the analysis of only LC1 (LC2). The probability at $\cos i=0$ was defined to be 0 . The Agol et al. (2009) estimate is based on models of the mid-IR SED and, according to the authors, should not be interpreted quantitatively.

(A color version of this figure is available in the online journal.)

which now depends relatively weakly on the black hole mass. The uncertainties in these estimates are logarithmic, corresponding to 0.4 and 0.3 dex, respectively, for Equations (3) and (6) if the masses, Eddington factors, luminosities, and efficiencies are viewed as being uncertain by a factor of 3 . We find that the disk is large compared to the estimate based on the observed flux, although this is modestly reduced by the inclination and small compared to a thin disk radiating close to Eddington with $\eta=10 \%$ efficiency. This is a discrepancy common to all microlensing estimates at present (see Pooley et al. 2007; Morgan et al. 2010).

Where these size estimates are incremental improvements over earlier results from using additional data and an improved physical model, our results for the inclination and orientation of the disk are entirely new. The preferred inclination is $\cos i>0.66$ at $68 \%$ confidence where $\cos i=1$ is face-on (Figure 4). Such a relatively face-on inclination is consistent with the expectations of the AGN unification model. We can compare this to Agol et al.'s (2009) model for the mid-infrared (mid-IR) spectral energy distribution (SED) of Q2237 using the dust torus models of Fritz et al. (2006). While Agol et al. (2009) do not trust the quantitative results, their preferred viewing angles $\left(i \leqslant 19^{\circ}\right.$, Figure 4$)$ are consistent with our inferences for the inclination angle of the disk.

Figure 5 shows our estimate of the P.A. of the major axis of the disk, and Figure 1 shows the 68\% confidence range of this P.A. for comparison to the anisotropies in the magnification patterns. We find consistent estimates of this P.A. from both LC1 and LC2 (Figure 5). The preferred orientation is for the major axis to be roughly parallel to the shear in image $\mathrm{B}$, but perpendicular to it in images C and D. Congdon et al. (2007) found that microlensing variability is enhanced when the major axis is aligned with the shear, so our estimated alignment helps to explain the higher variability of images $\mathrm{A}$ and $\mathrm{B}$, and the lower variability of $\mathrm{D}$, but not the variability of image $\mathrm{C}$. For this lens there are no 


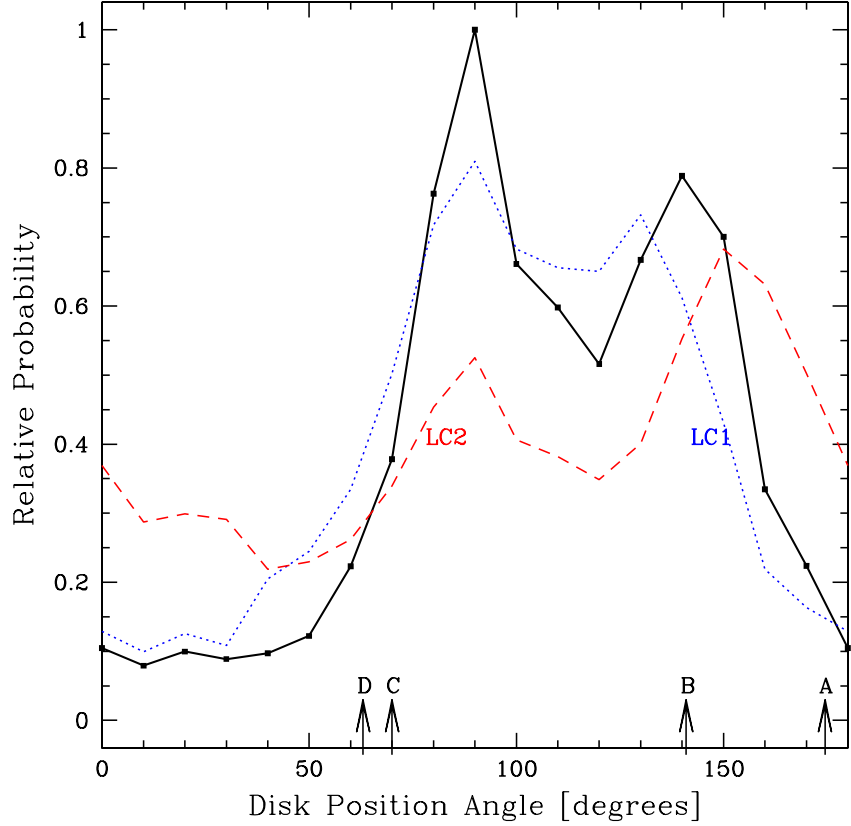

Figure 5. Major axis P.A. (North through East) of the accretion disk. The blue dotted (red dashed) curve shows the results from the analysis of only LC1 (LC2). The shear P.As for each image are indicated by the label arrows. The preferred orientation of the major axis is to be parallel to the shear of images A and B but perpendicular to $C$ and $D$. The face-on solutions are not included in these distributions because they provide no information on the P.A.

(A color version of this figure is available in the online journal.)

other observations to which we can compare the orientation. However, if we had observations of the quasar host galaxy, we could compare the P.A. of the galaxy to that of the disk as a check on the relative orientations of the angular momentum vectors of the accretion disk and the galaxy. For example, Yoo et al. (2005, 2006) used images of lensed quasar hosts to constrain the host axis ratio and P.A. in four lensed quasars (to roughly $\lesssim 20^{\circ}$ ). While both the peculiar velocity direction of the lens and the disk P.A. are constrained, we find no strong constraints on the disk orientation relative to the direction of motion (Figure 6). The convolved uncertainties in the two quantities are too large.

Luminosity that originates outside the accretion disk proper is a concern for size estimates because contamination by emission on large scales dilutes the microlensing signal and leads us to overestimate the projected area of the disk (see Dai et al. 2009; Morgan et al. 2008b). To examine this effect, we did a set of trials with $\left\langle M / M_{\odot}\right\rangle=0.3$, where $0 \%, 20 \%$, and $40 \%$ of the source light were not microlensed. The spectral analysis by Eigenbrod et al. (2008b) suggests that the level of contamination is $20 \%$. The $0 \%$ and $20 \%$ cases fit equally well, while the $40 \%$ case had a relative probability $38 \%$ lower. As the dilution is increased from $0 \%$, more face-on disks are preferred, with $\cos i>0.63,0.73$, and 0.80 (68\% confidence) for $0 \%, 20 \%$, and $40 \%$ dilution. Adding unmicrolensed light affects the disk size both through the general dilution and the shift toward more face-on orientations. The de-projected radius is smaller by $\sim 26 \%(55 \%)$ if the contamination is increased to $20 \%(40 \%)$ from $0 \%$. These corrections are not large compared to our statistical uncertainties of order $70 \%$, but they are an important physical consideration.

\section{DISCUSSION}

By including random stellar motions in our microlensing analysis of Q2237 we find evidence that the accretion disk of

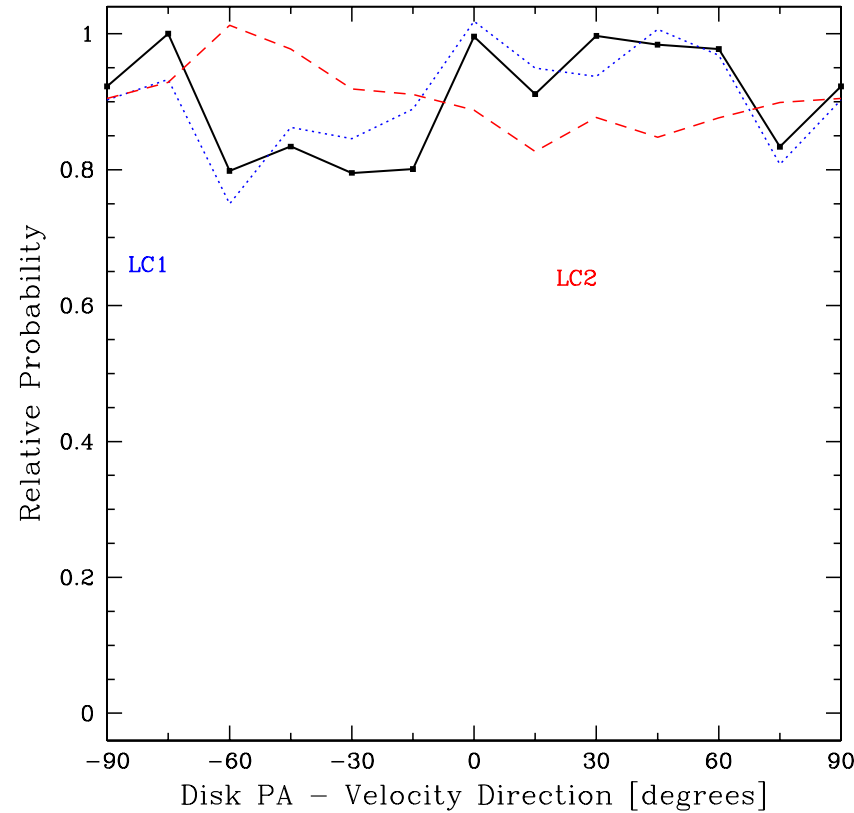

Figure 6. Major axis P.A. of the disk relative to the direction of motion (N through E P.As). Here we exclude the face-on trials since they add no useful information to this distribution.

(A color version of this figure is available in the online journal.)

Q2237 is viewed face-on with $\cos i>0.63$. This lends support to the popular AGN unification model where we expect Type 1 quasars like Q2237 to be viewed nearly face-on. Including $20 \%$ contamination from broad line emission on larger spatial scales (estimated from spectra Eigenbrod et al. 2008b) results in a stronger case for face-on solutions. Modeling stellar motions and the inclination and P.A. parameters further reduces the systematic uncertainties of our measurements of the disk radius compared to earlier studies by including a broader range of physical uncertainties. As we found in Paper I for the lens velocity and mean stellar mass, the results of the separate analyses of LC1 and LC2, the first and second temporal halves of the OGLE light curves, produce consistent results for every parameter we considered. While we have used a relatively simple model for the accretion disk, these results demonstrate that disk shapes can be measured with quasar microlensing, as suggested by Congdon et al. (2007). As data sets and computing power improve, it will be natural to try fitting more subtle disk features such as the asymmetries from relativistic effects using relativistic models such as Hubeny et al. (2001).

The exceptionally long OGLE light curve and the fast microlensing timescales of Q2337 made it a natural first choice for including the random stellar motions and studying the shape of the disk. Our expanded method is similar in computational cost to our previous efforts with static patterns, so there is no reason not to use it generally. The stellar motions clearly aid in reducing uncertainties in the mean mass (see Paper I) and allow us to correctly make inclination corrections. It can also easily be extended to take advantage of multi-wavelength data to try to constrain the temperature profile. However, as in earlier microlensing studies (Pooley et al. 2007; Morgan et al. 2010; Dai et al. 2009), we cannot reconcile the basic temperature profile of a thin disk, the microlensing size estimate, and the observed optical flux. A disk with a $T \propto R^{-3 / 4}$ temperature profile normalized by the microlensing size estimate should be brighter than observed. This can be solved by altering the 
temperature profile (see Poindexter et al. 2008; Morgan 2010). For example, reducing the slope from $T \propto R^{-3 / 4}$ to $T \propto R^{-1 / 2}$ would increase the flux size by a factor of 2.2 relative to the half-light radius. This would be mildly inconsistent $(1.5 \sigma)$ with the slope estimate of $-0.83 \pm 0.21$ by Eigenbrod et al. (2008a). However, such changes also call into question the basic structure of the thin disk model. The other simple possibility is to reduce the emissivity of the disk to be well below that of a blackbody (by the ratio of the flux/microlensing sizes squared), but this seems less physically plausible than change in the temperature structure.

This work was supported in part by an allocation of computing time from the Ohio Supercomputer Center. This research was supported by NSF grant AST-0708082.

\section{REFERENCES}

Agol, E. 1997, PhD thesis, Univ. of California, Santa Barbara

Agol, E., Gogarten, S. M., Gorjian, V., \& Kimball, A. 2009, ApJ, 697, 1010

Anguita, T., Schmidt, R. W., Turner, E. L., Wambsganss, J., Webster, R. L., Loomis, K. A., Long, D., \& McMillan, R. 2008, A\&A, 480, 327

Antonucci, R. 1993, ARA\&A, 31, 473

Bate, N. F., Floyd, D. J. E., Webster, R. L., \& Wyithe, J. S. B. 2008, MNRAS, 391,1955

Blaes, O. M. 2004, in Les Houches Summer School LXXVIII, ed. V. Beskin, G. Henri, \& F. Menard (Berlin: Springer), 137

Blandford, R. D., \& Konigl, A. 1979, ApJ, 232, 34

Chartas, G., Kochanek, C. S., Dai, X., Poindexter, S., \& Garmire, G. 2009, ApJ, 693, 174

Congdon, A. B., Keeton, C. R., \& Osmer, S. J. 2007, MNRAS, 376, 263

Dai, X., Kochanek, C. S., Chartas, G., Kozlowski, S., Morgan, C. W., Garmire, G., \& Agol, E. 2009, ApJ, 709, 278

Eigenbrod, A., Courbin, F., Meylan, G., Agol, E., Anguita, T., Schmidt, R. W., \& Wambsganss, J. 2008a, A\&A, 490, 933

Eigenbrod, A., Courbin, F., Sluse, D., Meylan, G., \& Agol, E. 2008b, A\&A, 480,647

Elvis, M. 2000, ApJ, 545, 63

Floyd, D. J. E., Bate, N. F., \& Webster, R. L. 2009, MNRAS, 398, 233
Fritz, J., Franceschini, A., \& Hatziminaoglou, E. 2006, MNRAS, 366, 767

Gould, A. 2000, ApJ, 535, 928

Hinshaw, G., et al. 2009, ApJS, 180, 225

Hubeny, I., Blaes, O., Krolik, J. H., \& Agol, E. 2001, ApJ, 559, 680

Huchra, J., Gorenstein, M., Kent, S., Shapiro, I., Smith, G., Horine, E., \& Perley, R. 1985, AJ, 90, 691

Kochanek, C. S. 2004, ApJ, 605, 58

Kochanek, C. S., Dai, X., Morgan, C., Morgan, N., Poindexter, S., \& Chartas, G. 2007, in ASP Conf. Ser. 371, Statistical Challenges in Modern Astronomy IV, ed. G. J. Babu \& E. D. Feigelson (San Francisco, CA: ASP), 43

Kollmeier, J. A., et al. 2006, ApJ, 648, 128

Koratkar, A., \& Blaes, O. 1999, PASP, 111, 1

Morgan, C. W. 2010, ApJ, in press (arXiv:1002.4160)

Morgan, C. W., Eyler, M. E., Kochanek, C. S., Morgan, N. D., Falco, E. E., Vuissoz, C., Courbin, F., \& Meylan, G. 2008a, ApJ, 676, 80

Morgan, C. W., Kochanek, C. S., Dai, X., Morgan, N. D., \& Falco, E. E. 2008b, ApJ, 689, 755

Morgan, C. W., Kochanek, C. S., Morgan, N. D., \& Falco, E. E. 2010, ApJ, in press (arXiv:1002.4160)

Mortonson, M. J., Schechter, P. L., \& Wambsganss, J. 2005, ApJ, 628, 594

Mosquera, A. M., Muñoz, J. A., \& Mediavilla, E. 2009, ApJ, 691, 1292

Poindexter, S., \& Kochanek, C. S. 2010, ApJ, 712, 658

Poindexter, S., Morgan, N., \& Kochanek, C. S. 2008, ApJ, 673, 34

Pooley, D., Blackburne, J. A., Rappaport, S., \& Schechter, P. L. 2007, ApJ, 661, 19

Shakura, N. I., \& Sunyaev, R. A. 1973, A\&A, 24, 337

Trott, C. M., Treu, T., Koopmans, L. V. E., \& Webster, R. L. 2010, MNRAS, 401,1540

Udalski, A., et al. 2006, Acta Astron., 56, 293

Urry, C. M., \& Padovani, P. 1995, PASP, 107, 803

Vakulik, V. G., Schild, R. E., Smirnov, G. V., Dudinov, V. N., \& Tsvetkova, V. S. 2007, MNRAS, 382, 819

Vestergaard, M., \& Peterson, B. M. 2006, ApJ, 641, 689

Wambsganss, J. 2006, in Gravitational Lensing: Strong Weak and Micro, SaasFee Advanced Course 33, ed. G. Meylan, P. North, \& P. Jetzer (Springer: Berlin), 453

Wyithe, J. S. B., Webster, R. L., \& Turner, E. L. 1999, MNRAS, 309, 261

Wyithe, J. S. B., Webster, R. L., \& Turner, E. L. 2000a, MNRAS, 315, 51

Wyithe, J. S. B., Webster, R. L., \& Turner, E. L. 2000b, MNRAS, 318, 762

Yee, H. K. C., \& De Robertis, M. M. 1991, ApJ, 381, 386

Yonehara, A. 2001, ApJ, 548, L127

Yoo, J., Kochanek, C. S., Falco, E. E., \& McLeod, B. A. 2005, ApJ, 626, 51

Yoo, J., Kochanek, C. S., Falco, E. E., \& McLeod, B. A. 2006, ApJ, 642, 22 